\title{
On the use of Indoor Radio Environment Maps for HetNets Deployment
}

\author{
A. Umbert, J.Pérez-Romero, F. Casadevall \\ Signal Theory and Communications Dept. \\ Universitat Politècnica de Catalunya (UPC) \\ Barcelona, Spain \\ \{annau | jorperez | ferranc\}@tsc.upc.edu
}

\author{
A. Kliks, P. Kryszkiewicz \\ Chair of Wireless Communications \\ Poznan University of Technology (PUT) \\ Polanka 3, 60-965 Poznań, Poland \\ \{akliks | pkrysz\}@et.put.poznan.pl
}

\begin{abstract}
This work analyses the feasibility of building an indoor Radio Environment Map (REM) for supporting the deployment of indoor small cells acting as secondary transmitters in the TVWS band. For that purpose, it proposes a structure of the REM database for computing the maximum allowed transmit powers not to interfere with TV receivers. The proposed framework is validated by means of real measurements. Results include the analysis of the spatial granularity and time stability of the measurements, and the maximum allowed transmit powers considering both co-channel and adjacent channel transmission, as well as the existence of other secondary transmitters. Results reveal that in the considered building small cell deployment using co-channel transmission is not feasible due to the very low resulting allowed transmit powers. However, when adjacent channel transmission is considered, the resulting maximum allowed power levels can be more adequate for successful small cells deployment.
\end{abstract}

\section{Keywords- TVWS, heterogeneous networks, indoor} measurements

\section{INTRODUCTION}

The exponential growth of the demand for mobile broadband services experienced in the last years associated with the massive penetration of the new generation of wireless user equipment (smartphones, tablets, etc.) in the population is expected to be continued in the future with the introduction of novel high bandwidth demanding applications such as High Definition Video, 3D, virtual reality, etc. For a better provision of such high capacity demanding service types the classical cellular network concept is being shifted towards the so-called Heterogeneous Networks (HetNets) composed of both traditional large macrocells and small cells of different sizes such as picocells, femtocells, etc. Such small cells are expected to cover small areas (in the order of 10-100m), including also indoor conditions, where it is envisaged that a significant part of this data traffic will be generated.

The massive introduction of small cells in dense urban scenarios will involve the use of efficient coordination mechanisms to reduce inter-cell interference. The use of different frequencies for the macrocells and for the small cells is also a possibility, at the expense of increasing the requirements in terms of spectrum demand. In this respect, the shared use of licensed frequency bands such as TeleVision White Spaces (TVWS) is seen as a relevant solution thanks to the more reduced coverage area and power levels of small cells, which makes easier the task of finding a sufficiently large TVWS to ensure the desired coverage. Different works in the literature have recognized recently the potentials of using TVWS in small cell scenarios [1]-[4], particularly for expanding LTE spectrum.

In this context, the potential of TVWS for deploying small cells in indoor scenarios is seen as particularly relevant because most of broadband data traffic is generated indoors, and also because it is expected that the availability of TVWS spectrum can be increased indoors thanks to the building penetration losses between indoor small cells and TV receiving antennas usually located at the rooftop or at least outside the building. In [5], some initial experimental studies based on indoor measurements were already carried out, validating a previous simulation model and identifying availability of TVWS in an indoor scenario. Also, in a prior work of the authors [6] the characteristics of the TVWS in indoor environments were analysed based on a measurement campaign to analyse the feasibility of creating Radio Environment Maps (REM) for guiding the operation of the small cells in the TVWS band. A REM is a knowledge database, constructed from spectrum measurements provided by different devices, used to dynamically store information related to the radio environment of wireless systems so that optimization in the use of spectrum resources can be achieved [7]. According to the different features of the obtained measurements in [6], such as the stability over time, the impact of the quotidian user mobility in the building or the effects of multi-path at different distances, it was concluded that the construction of such a REM based on measurements was feasible. Based on this prior work, this paper makes a step ahead by identifying the main parameters of an indoor REM and computing the allowed maximum transmit powers for the small cells in accordance with the obtained measurements and with the required protection ratios for different types of DVB-T (Digital Video Broadcast-Terrestrial) receivers.

This paper is organized as follows. Section II elaborates on the characteristics of the indoor REM database including the methodology to determinate, based on the available measurements, the allowed transmit power levels for secondary transmitters not to interfere the DVB-T receivers. Section III presents the measurement set-up to obtain the TVWS measurements, and Section IV presents the results on the allowed transmit power levels based on these measurements. Conclusions are summarised in Section V. 


\section{INDOOR REM DATABASE INFORMATION}

The REM should include all the relevant information needed to derive the transmit power limitations for any secondary transmitter (i.e. small cell) to be deployed in the building where the indoor REM applies or to modify the current transmission strategies by the secondary transmitters. Power limitations arise from the fact that the interference generated by the secondary transmitter to any DVB-T receivers should be below the acceptable limits not to degrade the TV reception capability.

Different works [8]-[10] have characterized the requirements for successful DVB-T reception. They are usually expressed in terms of the required Protection Ratio $(P R) . P R$ is defined as the minimum required ratio between the DVB-T signal and the interference at the DVB-T receiver input, i.e., it should be fulfilled that $P_{r} / I \geq P R$, where $P_{r}$ is the received DVB-T signal and $I$ is the total interference. $P R$ is also sometimes referred to as rejection threshold of Desired to Undesired received power $(\mathrm{D} / \mathrm{U})$. The $P R$ depends on whether the secondary transmits in the same channel as the DVB-T service and thus generates co-channel interference or whether it transmits in any of the adjacent channels. In the latter case, the $P R$ should account for the selectivity characteristic of the DVB-T receiver and for the ACLR (Adjacent Channel Leakage Ratio) of the secondary transmitter that determines how much of the transmitted power is actually transmitted in the adjacent channel. In the context of this work we assume that the secondary transmitter is an LTE base station (small cell) and we take as a reference the required $P R$ values that were obtained in [10] based on extensive measurements with different types of DVB-T receivers and LTE transmitters.

Under the above considerations, it is assumed that the indoor REM will contain a 3D characterisation of the radioelectrical propagation of the DVB-T signals inside the building as well as the required data to compute the maximum transmit powers. Specifically, the information to be stored can be classified as follows:

- Semi-static information: This corresponds to information elements that are not supposed to vary during secondary system operation, so that they can be acquired at some point of time and be valid for a long period of time as long as regulatory conditions do not change (i.e. no new DVB-T licenses are assigned) nor new DVB-T transmitters appear. In particular, the following elements are considered:

- $P_{r}(\theta, N)$ : Received power level of the DVB-T signal at each position $\theta=(x, y, z)$ inside the building for each TV channel $N$

- $P R(i)$ : Required protection ratio to ensure DVB-T reception in channel $N$ when the secondary transmitter is working at channel $N+i$. Note that the case $i=0$ corresponds to the co-channel protection ratio while the case $i>0$ corresponds to the $i$-th adjacent channel protection ratio.

- $P_{r, \min }$ : Minimum received power level to ensure successful DVB-T reception.

- $L(d)$ : Indoor propagation model to characterise the losses between any two points of the building as a func- tion of the distance $d$ and the building characteristics (e.g. number of floors, etc.).

- Dynamic information: This corresponds to information that needs to be updated dynamically depending on the operation of the secondary system. The following information is considered:

- $P_{\operatorname{Tmax}}(\theta, N)$ : Maximum allowed transmit power for a secondary transmitter located at position $\theta=(x, y, z)$ and operating on TV channel $N$. This maximum power level will depend on the $P R$ requirements of the DVB-T receivers and on the number and positions of the currently active secondary transmitters inside the building, in order to ensure that the aggregated interference that they generate is below the acceptable limits established by the $P R$. Correspondingly, this information needs to be updated every time that a new small cell is activated or deactivated in the considered building.

The focus of this paper will be mainly placed on the acquisition of the received power levels $P_{r}(\theta, N)$ by means of measurements made with a certain granularity, as it will be discussed in Section IV. Concerning $P R$ and $P_{r, \min }$ the values from [10] will be retained. As for the indoor propagation, the model from [11] is considered with the floor characteristics of the building. It is worth mentioning that the indoor propagation model could also be obtained based on measurements, but this is left for future work.

In order to compute the maximum allowed transmit power this paper will focus on the worst case scenario in which the positions of the DVB-T receivers are unknown (e.g. in case of USB-stick DVB-T receivers connected to laptops that can be in any part of the building). However, in indoor scenarios other situations could also be considered in which e.g. the positions of the at least static DVB-T receivers could be registered in the database as well, or in which the only DVB-T reception point is the antenna at the rooftop.

\section{A. Maximum allowed transmit power in the absence of other secondary transmitters}

Assuming there is no other secondary transmitter in the building, the maximum allowed transmit power at point $\theta=(x, y, z)$ for a general channel $N+i$ needs to fulfil the following condition for any point $\theta$ ' where a DVB-T receiver could be located:

$$
\frac{P_{r}\left(\theta^{\prime}, N\right)}{\frac{P_{T \max }(\theta, N+i)}{L\left(\theta, \theta^{\prime}\right)}} \geq P R(i)
$$

where $L\left(\theta, \theta^{\prime}\right)$ denotes the propagation losses between the point $\theta$ where the secondary transmitter is located and the point $\theta$ ' where the potential DVB-T receiver could be located. Based on this relationship the following distinction is done:

\section{1) Co-channel secondary transmission $(i=0)$}

In this case, it will be assumed that no secondary transmission is allowed at point $\theta$ if DVB-T reception is possible at this point (i.e. if $\left.P_{r}(\theta, N) \geq P_{r, m i n}\right)$. Though this condition would not be strictly necessary in theory as long as the protection ratio $P R(0)$ constraint is ensured when determining $P_{\text {Tmax }}$, in practice this would lead to very small values of transmit power 
that would actually prevent the secondary transmission. For this reason, we restrict secondary transmission to the points $\theta$ where DVB-T reception is not possible. In this case, the maximum transmit power is obtained from the most restrictive of the points $\theta$ ' where DVB-T reception is possible. Then, from (1) it is obtained that:

$$
P_{T \max }(\theta, N)= \begin{cases}0 & \text { if } P_{r}(\theta, N) \geq P_{r \min } \\ \min _{\theta^{\prime} \text { s.t. } P_{r}\left(\theta^{\prime}, N\right) \geq P_{\text {min }}}\left[\frac{P_{r}\left(\theta^{\prime}, N\right) \cdot L\left(\theta, \theta^{\prime}\right)}{P R(0)}\right] & \text { if } P_{r}(\theta, N)<P_{r \min }\end{cases}
$$

\section{2) Adjacent channel secondary transmission ( $i>0)$ :}

The main difference with the previous case is that now, since $P R(i)$ values are much lower than in the co-channel case, transmission in the adjacent channel $N+i$ can be considered even at a point where the DVB-T reception in channel $N$ is possible. Then, from (1) the maximum transmit power is given by:

$$
P_{T \text { max }}(\theta, N+i)=\min _{\theta^{\prime} \text { s.t. } P_{r}\left(\theta^{\prime}, N\right) \geq P_{r \min }}\left[\frac{P_{r}\left(\theta^{\prime}, N\right) \cdot L\left(\theta, \theta^{\prime}\right)}{P R(i)}\right]
$$

For the case $\theta=\theta$ ' in which the secondary transmitter and the DVB-T receiver are located in the same position, it is assumed that in practice there will be always a minimum physical separation between the secondary transmitter and the DVB-T receiver [12], so $L\left(\theta^{\prime}, \theta^{\circ}\right)$ equals a minimum propagation loss $L_{\min }$ related with this physical separation.

B. Maximum allowed transmit power when there are other secondary transmitters

In case that a secondary transmitter already exists at a certain position $\theta^{*}$ operating in channel $N+i$ with power $P_{T s e c}$ (where $P_{\text {Tsec }}$ should be lower than $P_{\text {Tmax }}\left(\theta^{*}, N+i\right)$ computed from (2) and (3)), the maximum allowed power levels $P_{\text {Tmax }}(\theta, N)$ stored in the REM need to account for the secondary interference already present in the building. In particular, if another transmitter had to be placed at position $\theta \neq \theta^{*}$, the following condition has to be fulfilled for any point $\theta$ ' where the DVB-T receiver can be located:

$$
\frac{P_{r}\left(\theta^{\prime}, N\right)}{\frac{P_{T \mathrm{sec}}}{L\left(\theta^{*}, \theta^{\prime}\right)}+\frac{P_{T \max }(\theta, N+i)}{L\left(\theta, \theta^{\prime}\right)}} \geq P R(i)
$$

Based on this expression, the maximum allowed transmit power can be formulated in a similar way as in (2) and (3):

\section{1). Co-channel secondary transmission $(i=0)$ :}

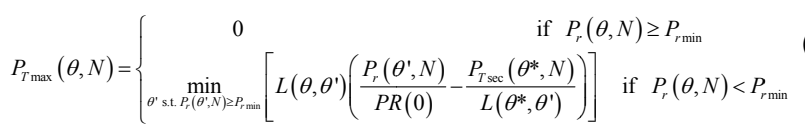

\section{2). Adjacent channel secondary transmission $(i>0)$.}

$$
P_{T_{\max }}(\theta, N+i)=\min _{\theta^{\prime} \text { s.t. } P_{r}\left(\theta^{\prime}, N\right) \geq P_{r \min }}\left[L\left(\theta, \theta^{\prime}\right)\left(\frac{P_{r}\left(\theta^{\prime}, N\right)}{P R(i)}-\frac{P_{T_{\text {sec }}}\left(\theta^{*}, N+i\right)}{L\left(\theta^{*}, \theta^{\prime}\right)}\right)\right]
$$

Computations could easily be extended to consider the case with multiple existing secondary transmitters. Note in any case that the above computations assume that no modification is made in the transmit power level of the already existing secondary transmitter. Another possibility, left for future work, would be to perform an optimization process to jointly adjust the power of all the secondary transmitters whenever a new one has to be added.

It is worth mentioning that the analysis made in this paper assumes that there is sufficient isolation between different buildings to consider that a secondary transmitter located in one building does not interfere to DVB-T receivers in other buildings. While this is a simplification that needs to be further assessed with new measurements, it allows having a first estimation of the allowed transmit power levels and their feasibility to deploy indoor small cells.

\section{MEASUREMENT LOCATION AND SET-UP}

The TVWS measurements to generate the indoor REM power levels $P_{r}(x, y, z, N)$ discussed in the previous section have been obtained in building D4 of the UPC Campus Nord in Barcelona. The location of this building is latitude: $41^{\circ} 23^{\prime} 20^{\prime \prime}$ $\mathrm{N}$; longitude: $2^{\circ} 6^{\prime} 43^{\prime \prime} \mathrm{E}$; altitude: $175 \mathrm{~m}$. A total of $21 \mathrm{DVB}-$ $\mathrm{T}$ channels were detected at the rooftop. Although different channels were measured, for the sake of simplicity and to get a first insight on the potential allowed transmit power levels for secondary transmission, the results presented in this paper focus only DVB-T channel $61(794 \mathrm{MHz})$. The channel is received from a TV tower transmitter (named Torre de Collserola, see Fig. 1) located at $3.1 \mathrm{~km}$ with transmit power of $7.4 \mathrm{~kW}$ for this channel.

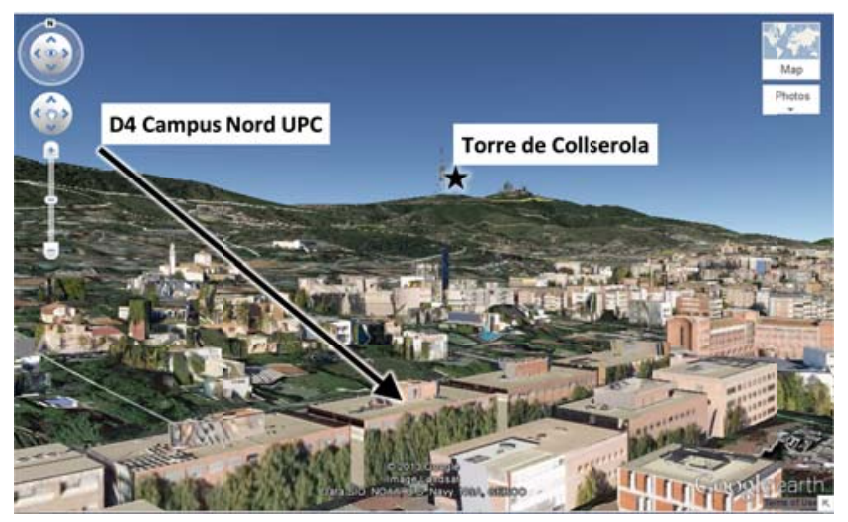

Fig. 1. Position of TV transmitter and the measured building.

The DVB-T signal has been measured by means of an omnidirectional antenna, then sent to a spectrum analyzer and finally stored on a portable computer via Matlab. The system setup is shown in Fig. 2.
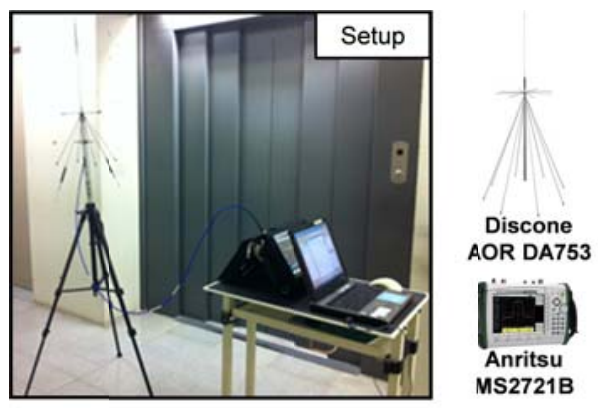

Fig. 2. Measurements system setup. 
A passive discone antenna of type AOR DN753 was used, covering the frequency range from 75 to $3000 \mathrm{MHz}$, and connected to ANRITSU MS2721B spectrum analyser. The resolution and video bandwidth of the spectrum analyzer are $\mathrm{RBW}=30 \mathrm{kHz}$ and $\mathrm{VBW}=100 \mathrm{kHz}$, respectively.

\section{MEASUREMENT ANALYSIS}

\section{A. Spatial granularity and stability analysis}

First results of this section intend to analyse the required spatial granularity for the measurements stored in the indoor REM. For that purpose, multiple measurements have been performed in different positions inside the considered building. It is a four-level building with roughly $420 \mathrm{~m}^{2}$ per floor (30m $\times 14 \mathrm{~m})$ including basement, ground floor, first and second floors. As an example, Fig. 3 shows the plan of the first floor where it can be observed how it is split in different offices/rooms. Measurements focus mainly in the interior part of the rooms where it can be envisaged that DVB-T receivers can be located, though some points in the corridors are also considered. Rooms are classified into small size offices (around $3 \mathrm{~m} \times 4 \mathrm{~m}$ ), medium size offices (e.g. those located in the four corners of Fig. 3) and large size rooms (e.g. laboratory D4-115 indicated in Fig. 3).

To assess the differences in received power inside the small size offices, initially 2 measurement points per office were considered located at two positions separated around $2 \mathrm{~m}$, one located close to the window and the other close to the internal corridor. Table 1 presents the results in these two points for three offices indicated in Fig. 3. In each point a total of 50 measurements of the power level received in the $8 \mathrm{MHz}$ bandwidth of the DVB-T channel have been done. Presented results include the average and the standard deviation. Results in Table 1 reveal that the differences between the two points are relatively small, between 1 and $2 \mathrm{~dB}$. Moreover, also low values of standard deviation can be observed.

Based on these observations, it was decided that one single point of measurement was sufficient for small size offices, while for large/medium size rooms measurements were made in more granularity, leading to the measurement points shown in Fig. 3 for the case of the first floor. Similar distributions of measurements were also made for the other floors.

Table 1. Measurements done in small offices at two points, one close to the window and the other close to the internal corridor

\begin{tabular}{|c|c|c|c|c|}
\hline \multirow{2}{*}{ Office } & \multicolumn{2}{|c|}{ Window Point } & \multicolumn{2}{c|}{ Corridor Point } \\
\cline { 2 - 5 } & $\begin{array}{c}\text { Average } \\
(\mathrm{dBm})\end{array}$ & $\begin{array}{c}\text { StDev } \\
(\mathrm{dB})\end{array}$ & $\begin{array}{c}\text { Average } \\
(\mathrm{dBm})\end{array}$ & $\begin{array}{c}\text { StDev } \\
(\mathrm{dB})\end{array}$ \\
\hline D4-105 & -60.54 & 0.73 & -59.44 & 0.29 \\
\hline D4-106 & -53.02 & 0.57 & -54.30 & 0.98 \\
\hline D4-108 & -60.45 & 0.94 & -62.67 & 0.86 \\
\hline
\end{tabular}

To assess the time stability of indoor measurements, the variation of the measured signal in specific points at different time instants was analysed. A first result in this respect is shown in Table 1, where the presented standard deviation for each point corresponds to a measurement duration of around $10 \mathrm{~min}$. The low values of standard deviation obtained, below 1 $\mathrm{dB}$, suggest that a good stability is obtained for short term periods in the order of minutes. Moreover, in order to analyse the stability over long periods of time, measurements in some selected points were also repeated in different days and times. As an illustrative example of these measurements, in the case of the point inside laboratory D4-115 labelled A of Fig. 3, a total of 9 measurements done in 3 days and 3 different times of the day revealed that the standard deviation is only $2.11 \mathrm{~dB}$. Consequently, it was considered that the time stability of the obtained measurements was sufficiently good so that measurements can be stored in an indoor REM. Similar observations in this respect where obtained in the prior work [6].

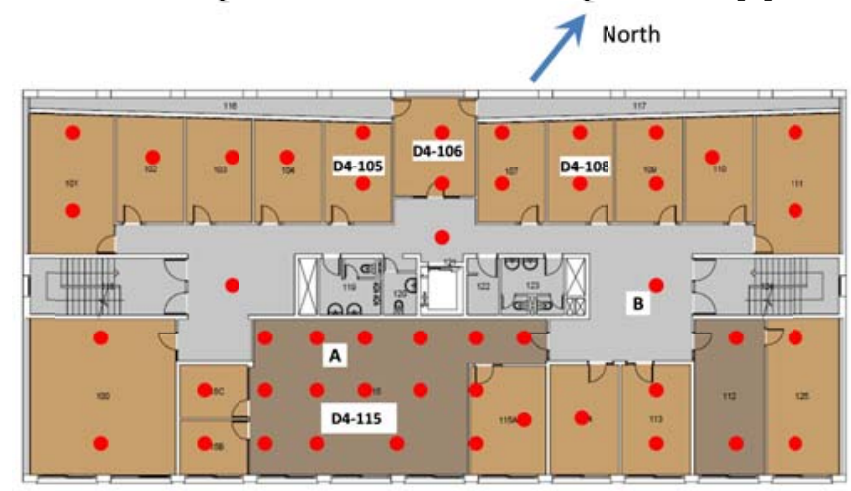

Fig. 3. First floor measurement points in the building.

\section{B. Maximum Transmit Power when there is no other secondary transmitter}

This section evaluates the dynamic information stored in the REM as indicated in Section II. In particular, it computes the maximum transmit power that can be allowed in the different points of the building assuming that there is no other active secondary transmitter. For that purpose, expressions (2) and (3) were used to determine $P_{\operatorname{Tmax}}$ for all the points of the building considering co-channel transmission in channel $N=61$ and adjacent channel transmission in $N+1$ and $N+2$.

The minimum received field strength to ensure successful reception of the DVB-T signal is assumed to be $56.7 \mathrm{~dB} \mu \mathrm{V} / \mathrm{m}$ [10], which corresponds to $P_{r, \min }=-80.5 \mathrm{dBm}$. The required protection ratios $P R(i)$ are obtained assuming two different types of receivers, namely an USB-Stick DVB-T receiver to be connected to PCs and laptops and a TV set with integrated digital tuner (IDTV) [10]. The indoor propagation losses are computed using the model adopted by FemtoForum and 3GPP TSG RAN4 [11] for communication between a terminal and a femtocell inside the same house. A frequency correction term to adapt the model to the TV band was introduced. Moreover, the minimum propagation loss existing between the secondary transmitter and any DVB-T receiver assuming that there will always be some physical separation between the two is assumed to be $L_{\min }=35 \mathrm{~dB}$, that results from considering a distance of $2 \mathrm{~m}$ in the propagation model.

Focusing first on the possibility of having co-channel transmission in channel $N=61$, given that the building has direct line-of-sight and short distance with the TV tower transmitter, DVB-T reception is possible in most of the points inside the building, which prevents from the possibility of doing co-channel transmission in most of the cases. The only exceptions are a few points located at the basement, which is located below the street level. These points represent only $7.23 \%$ of the points in the building. Table 2 presents the $P_{\text {Tmax }}$ 
statistics for these points according to (2) for the two types of DVB-T receivers considered (USB stick and IDTV). It is observed that the maximum allowed transmit power takes actually very small values that would limit the coverage of the secondary transmitter to very few meters. In this respect it is not envisaged that these values could allow for the deployment of a small cell to provide satisfactory coverage in different parts of the building.

Table 2. $\mathrm{P}_{\mathrm{Tmax}}$ for co-channel transmission in $\mathrm{N}=61$

\begin{tabular}{|c|c|c|}
\hline $\mathrm{P}_{\text {Tmax }}$ & USB Stick & IDTV \\
\hline Min & $-51.95 \mathrm{dBm}$ & $-50.95 \mathrm{dBm}$ \\
\hline Avg & $-46.59 \mathrm{dBm}$ & $-45.59 \mathrm{dBm}$ \\
\hline Max & $-39.50 \mathrm{dBm}$ & $-38.50 \mathrm{dBm}$ \\
\hline
\end{tabular}

Concerning the secondary transmission in adjacent channels, Fig. 4 plots the Cumulative Distribution Function (CDF) of the maximum allowed transmit power $P_{\text {Tmax }}$ according to (3) for the different points inside the building. Results are presented for both adjacent channels $N+1$ and $N+2$ and for both types of DVB-T receivers. As it can be observed, the allowed power levels are substantially higher than in the co-channel case, so it can be feasible the potential deployment of a small cell acting as a secondary transmitter. Specifically, when considering the USB-Stick DVB-T receiver, $90 \%$ of the points have a maximum allowed transmit power between $-12 \mathrm{dBm}$ and $9 \mathrm{dBm}$ in the first adjacent channel, and between $-10 \mathrm{dBm}$ and $11 \mathrm{dBm}$ in the second. Moreover, in half of the points the transmit power can be higher than $-6 \mathrm{dBm}$ in the first adjacent channel, which can be assumed a feasible level for successfully deploying an indoor small cell. For the IDTV receiver these values increase in around $2 \mathrm{~dB}$.

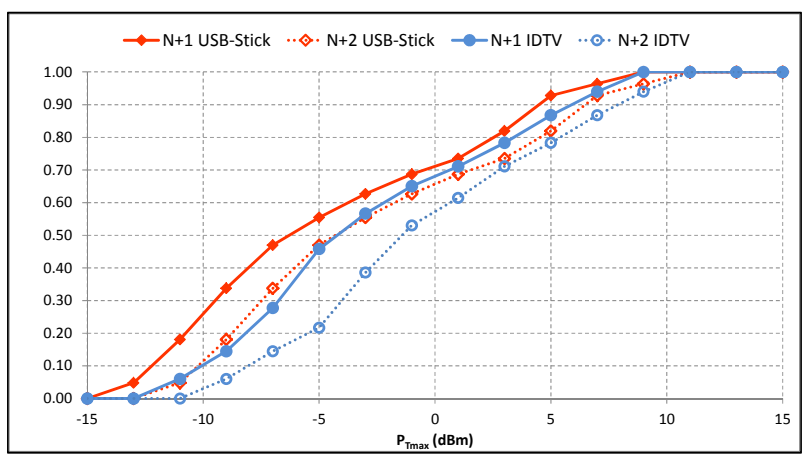

Fig. 4. CDF of the maximum transmit power $\mathrm{P}_{\text {Tmax }}$ for first and second adjacent channels

To gain insight on how the maximum power is distributed in different parts of the building, Table 3 presents different statistics of $P_{T \max }$ for different floors. Results are presented for the 1 st adjacent channel and the USB stick receiver. In the case of the 1st floor measurements, positions have been further split between those lying in the northern and southern frontage sides (see Fig. 3) and between eastern and western sides. In that respect, the northern and eastern frontages have better visibility conditions with respect to the TV transmit tower than the western and southern frontages. Therefore, it can be observed in the table that the maximum allowed power levels for the points located in the northern and eastern sides are larger than in the southern/western sides, because the DVB-T receiv- er at northern/eastern points has better signal conditions. It can also be noticed in the table that the maximum allowed transmit power tends to decrease when considering the ground floor and the basement.

Table 3. $\mathrm{P}_{\text {Tmax }}$ statistics for adjacent channel $\mathrm{N}+1$ in the different floors

\begin{tabular}{|c|c|c|c|}
\cline { 2 - 4 } \multicolumn{1}{c|}{} & $\begin{array}{c}\text { Average } \\
(\mathrm{dBm})\end{array}$ & $\begin{array}{c}\text { 5-th Percentile } \\
(\mathrm{dBm})\end{array}$ & $\begin{array}{c}\text { 95-th Percentile } \\
(\mathrm{dBm})\end{array}$ \\
\hline Basement & -7.47 & -13.61 & 0.15 \\
\hline Ground floor & -3.06 & -10.94 & 5.70 \\
\hline 1st floor (south side) & -9.15 & -12.68 & -4.48 \\
\hline 1st floor (north side) & 3.40 & -0.21 & 8.95 \\
\hline 1st floor (east side) & -3.20 & -11.09 & 8.84 \\
\hline 1 st floor (west side) & -5.48 & -12.68 & 3.72 \\
\hline
\end{tabular}

\section{Maximum Transmit Power when there is another secondary transmitter}

This subsection analyses the case when there already exists a secondary transmitter in the building and the indoor REM has to be recomputed in order to determine the new maximum allowed power levels in each position according to (5)(6). Specifically, the existing secondary transmitter is assumed to be in the 1st floor at location labeled B (see Fig. 3). Considering adjacent channel $N+1$ and USB stick receiver, the maximum allowed transmit power at this location in order not to interfere with the DVB-T receivers would be $-3.2 \mathrm{dBm}$. Accordingly, the transmit power of this secondary transmitter $P_{T s e c}$ has been set to three possible values, namely $-3.5 \mathrm{dBm}$, $-5 \mathrm{dBm},-10 \mathrm{dBm}$. It is worth mentioning that the study only focuses on the interference that the secondary transmitters cause to the DVB-T receivers, so the mutual interference between secondary users is not taken into account. The considerations about this mutual interference are left for future work.

Under the abovementioned conditions, Fig. 5 plots the $\mathrm{CDF}$ of the new values for the maximum transmit power $P_{\text {Tmax }}$ in the different positions inside the building for the above set of power levels and also for the case when there is no other secondary transmitter. It can be observed that $P_{T \max }$ values decrease when increasing the power $P_{T s e c}$ of the secondary transmitter. However, only when $P_{\text {Tsec }}$ is $-3.5 \mathrm{dBm}$ (i.e. very close to the maximum allowed power level of $-3.2 \mathrm{dBm}$ ), significant differences are observed in the CDF. Differences are particularly noticeable for the largest values of $P_{\text {Tmax }}$ which are between 2 and $3 \mathrm{~dB}$ less than when there is no other secondary transmitter.

The reason for such small differences is that the already existing secondary transmitter only has influence over a relatively small part of the building. To further analyse this fact, Table 4 presents the $P_{\operatorname{Tmax}}$ statistics in different parts of the building when the transmit power of the existing secondary transmitter is $P_{T s e c}=-3.5 \mathrm{dBm}$. By comparing the results in Table 4 with those in Table 3 where no other secondary transmitter exists, it can be observed that the influence of the existing secondary transmitter in the first floor is negligible at the points in the ground floor and the basement. In turn, for the points in the first floor differences are particularly remarkable when considering the points at the northern side $(6 \mathrm{~dB}$ reduction in the 95-th percentile) and at the eastern side $(10 \mathrm{~dB}$ 
reduction in the 95-th percentile) that correspond to the area closer to the position of the existing secondary transmitter (point B in Fig. 3). On the contrary, for the points in the southern and western sides differences are much smaller (in the order of $1.3 \mathrm{~dB}$ for the 95 -th percentile).

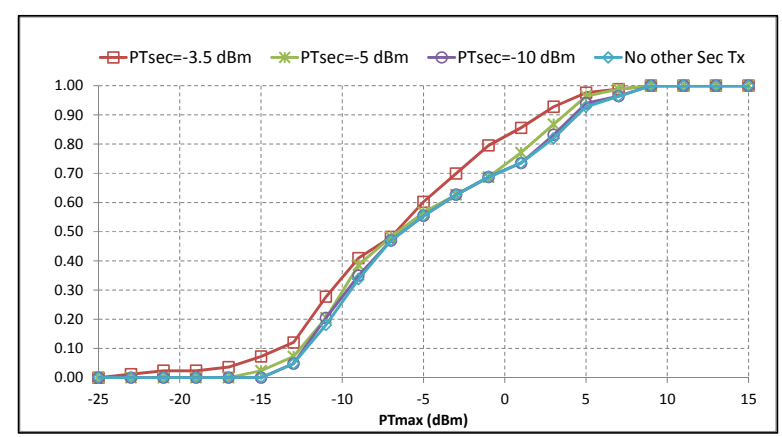

Fig. 5. CDF of the maximum transmit power $\mathrm{P}_{\mathrm{Tmax}}$ for first adjacent channels when there is another secondary transmitter

Table 4. $\mathrm{P}_{\text {Tmax }}$ statistics for adjacent channel $N+1$ in the different floors when there is another secondary transmitter in the $1 \mathrm{st}$ floor with $\mathrm{P}_{\mathrm{Tsec}}=-3.5 \mathrm{dBm}$

\begin{tabular}{|c|c|c|c|}
\cline { 2 - 4 } \multicolumn{1}{c|}{} & $\begin{array}{c}\text { Average } \\
(\mathrm{dBm})\end{array}$ & $\begin{array}{c}\text { 5-th Percentile } \\
(\mathrm{dBm})\end{array}$ & $\begin{array}{c}\text { 95-th Percentile } \\
(\mathrm{dBm})\end{array}$ \\
\hline Basement & -7.47 & -13.61 & 0.15 \\
\hline Ground floor & -3.06 & -10.94 & 5.70 \\
\hline 1st floor (south side) & -11.61 & -20.71 & -5.81 \\
\hline 1st floor (north side) & -1.36 & -5.58 & 2.88 \\
\hline 1 st floor (east side) & -9.87 & -22.62 & -1.25 \\
\hline 1st floor (west side) & -5.91 & -12.81 & 2.47 \\
\hline
\end{tabular}

\section{CONCLUSIONS}

This work has analysed the feasibility of building an indoor REM for supporting the deployment of indoor small cells operating as secondary transmitters in the TVWS band. In this respect, the paper has proposed the structure of the REM database with the most relevant information for computing the maximum allowed transmit powers not to interfere with the primary DVB-T receivers existing in a building.

The proposed indoor REM framework has been validated making use of real measurements in a University building. Results have addressed first the spatial granularity and time stability aspects of the measurements stored in the REM. It has been obtained that for small size offices a single point of measurement was sufficiently representative while a larger granularity was required for medium size and large size (labs) offices. Standard deviation of the measured signals in different days has been in the order of $2 \mathrm{~dB}$, revealing that the time stability of the obtained measurements was sufficiently good so that they can be stored in an indoor REM.

Results have also analysed the maximum allowed transmit powers for secondary transmitter considering the protection ratio characteristics of different types of receivers. It has been obtained that the deployment of a small cell acting as a cochannel secondary transmitter is in practice not possible in the considered building, because of the very low maximum allowed transmit powers that would prevent from having satisfactory small cell coverage. On the contrary, when considering adjacent channel transmission and USB-stick DVB-T receivers, it has been obtained that in half of the points inside the building the transmit power can be higher than $-6 \mathrm{dBm}$ in the first adjacent channel, which can be assumed a feasible level for successfully deploying a small cell. The maximum transmit powers increase in around $2 \mathrm{~dB}$ when considering an IDTV receiver. The paper has also analysed the differences in the statistical distribution of the maximum allowed transmit power in different parts of the building considering also the presence of another secondary transmitter. In this respect, it has been obtained that the impact of this transmitter is mainly on the surroundings of its location, but it has little impact on the allowed transmit power for transmitters in e.g. other floors.

Based on these results, future work includes the analysis of the impact towards DVB-T receivers located in other buildings close to the considered one, the analysis of other DVB-T channels and the optimisation of the maximum allowed transmit power when multiple small cells exist. This includes also the study of achievable coverage and capacity for the small cell deployments.

\section{ACKNOWLEDGMENT}

This work has been supported by FP7 NEWCOM\# project (grant number 318306) and by the Spanish Research Council and FEDER funds under ARCO grant (ref. TEC2010-15198).

\section{REFERENCES}

[1] J. Xiao, R. Q. Hu, Y. Qian, L. Gong, B. Wang "Expanding LTE Network Spectrum with Cognitive Radios: From Concept to Implementation", IEEE Wireless Comms, April, 2013, pp. 13-19

[2] J. Zander, L.K. Rasmussen, K.W. Song, P. Mähönen, M. Petrova, R. Jäntti, J. Kronander, "On the scalability of Cognitive Radio: Assessing the Commercial Viability of Secondary Spectrum Access", , IEEE Wireless Communications, April, 2013, pp.28-35

[3] T. Dudda, T. Irnich, "Capacity of cellular networks deployed in TV White Space", IEEE International Symposium on Dynamic Spectrum Access Networks, 2012.

[4] A. Achtzehn, M. Petrova, P. Mähönen, "On the Performance of Cellular Network Deployments in TV Whitespaces", IEEE ICC 2012.

[5] E. Obregon, L. Shi, J. Ferrer, J. Zander, "Experimental Verification of Indoor TV White Space Opportunity Prediction Model", CROWNCOM, 2010.

[6] A. Kliks, P. Kryszkiewicz, A. Umbert, J.Pérez-Romero, F. Casadevall, "TVWS Indoor measurements for HetNets", Workshop on Interference and Design Issues in Future Heterogeneous Networks at IEEE WCNC conference, Istambul, April, 2014.

[7] Y. Zhao, L. Morales, J. Gaeddert, K. K. Bae, J.-S. Um, and J. H. Reed, "Applying radio environment maps to cognitive wireless regional area networks," DySPAN07, Dublin, Ireland, April, 2007.

[8] Advanced Television Systems Committee "ATSC Recommended Practice: Receiver Performance Guidelines (with Corrigendum No. 1 and Amendment No.1)", Doc. A/74, November, 2007.

[9] G. L. Stüber, S. M. Almalfouh, D. Sale, "Interference Analysis of TVBand Whitespace", Proceedings of the IEEE, Vol. 97, No. 4, April, 2009, pp. 741-754.

[10] H. Aïache, et al. "Use-cases Analysis and TVWS Systems Requirements", Deliverable D3.1 of the COGEU project, August, 2010.

[11] 3GPP TSG RAN WG4 Meeting \#51: R4-092042 "Simulation assumptions and parameters for FDD HeNB RF requirements", AlcatelLucent, May 2009.

[12] J. Lauterjung et al. "Spectrum measurements and anti-interference spectrum database specification", Deliverable D4.1 of the COGEU project, October, 2010 